An important class of applications of measurement error or constrained factor analytic models consists of comparing models for several populations. In such cases it is appropriate to make explicit statistical tests of model similarity across groups and to constrain some model parameters to be equal across groups using a priori substantive information. This article discusses a statistical model developed by Jöreskog for these purposes. The model is applied to children's and parents' reports of parental socioeconomic statuses for several grade levels.

\title{
Children's Reports of \\ Parental Socioeconomic Status
}

A Multiple Group Measurement Model

\author{
ROBERT D. MARE \\ University of Wisconsin-Madison \\ WILLIAM M. MASON \\ University of Michigan
}

$1 / 7$

uch recent research has been concerned with measurement error and its implications for linear models of sociological phenomena. The literature is now rich with attempts to model and estimate measurement error and to use the resulting models to construct more sophisticated structural equation models (Goldberger and Duncan, 1973; Bielby and Hauser, 1977b). Within this framework, an important class of applications consists of intergroup comparisons of measurement or constrained factor analytic models relating observable indicators to unobservable latent variables. This may consist of estimating a

\footnotetext{
AUTHORS' NOTE: We are indebted to Alan C. Kerckhoff for making available to us the data used in this article. This research was supported in part by funds granted to the Institute for Research on Poverty at the University of WisconsinMadison by the Department of Health, Education, and Welfare pursuant to the provisions of the Economic Opportunity Act of 1964.
} 
measurement or factor model for the same population at two points in time, or of comparing the models for several populations at a point in time. For example, K. O. Mason et al. (1976) examine change in sex-role attitude factor models between the early 1960 s and the mid-1970s. Bielby et al. (1977a) estimate response error models for measures of socioeconomic attainment in black and nonblack populations. And W. M. Mason et al. (1976) compare the accuracy of children's reports of parental socioeconomic statuses among age-race groups. In each case separate models of the relationships between unobserved and observed variables and among the unobserved variables themselves are specified for the groups of interest. The pattern and the strength of these relationships, both within and across populations, are central substantive concerns.

Thus far, most comparisons of measurement error and factor analytic models for two or more groups have been deficient because they estimated models independently for each group. That is, they have lacked a common framework within which to estimate the models for all groups simultaneously. For example, in contrasting the response error patterns of children of different age groups, W. M. Mason et al. (1976) estimate separate models for each group, allowing all group differences to be reflected in the parameter estimates. In general, this procedure has two important limitations. First, it precludes statistical tests of group differences in the measurement models. A test for group differences requires a comparison of two models: (1) a model in which the specifications (or parts of them) for the several groups are constrained to be the same; and (2) an unconstrained model in which the specifications (or parts of them) vary across groups. The second model always fits the data better than the first because it has more parameters. The test consists of assessing the statistical significance of the improvement in fit in going from the first model to the second. The test requires a framework for, first, obtaining pooled (constrained) estimates for the several groups and, second, estimating the unconstrained model for all groups simultaneously. Estimating separate models for each 
group does not permit the explicit comparison required by the test.

A second limitation of estimating the models of each group independently from the others is that it fails to exploit intergroup similarity in measurement models, resulting in less reliable parameter estimates than would otherwise be possible. Groups may differ in part of their measurement error pattern but be invariant in others. For example, the correlations between attitude constructs may change over time, but the relationships between attitude constructs and specific response items may not. A reasonable strategy, then, is to hold constant some elements of the models and allow variation where substantive reasoning indicates that it may occur. This affords better parameter estimates because they are based on more observations (a result of pooling groups) and use fewer degrees of freedom (a result of between-group equality constraints).

There is a statistical framework for the simultaneous estimation of measurement models in several populations (Jöreskog, 1971a). ${ }^{1}$ To date, however, it has received little use in social research, despite the common task of comparing measurement or restricted factor analytic models across groups. We intend, in this article, to illustrate the use of Jöreskog's framework through a substantive example; namely, age variation in the reliability of children's reports of parental socioeconomic characteristics. We will analyze the data drawing upon the substantive arguments of W. M. Mason et al. (1976) and refining that earlier work. Our article will, first, review the problem of response unreliability in children's reports of parental characteristics and, second, will present a measurement model for children's and parents' reports for a single population, discussing its weaknesses. Third, it will outline a multiple-group approach to measurement error estimation and apply Jöreskog's (1971a) framework to the substantive problemi at hand. Fourth, it will discuss alternative specifications of the measurement model in connection with specific hypotheses. Finally, it will present empirical results. 


\section{ERRORS IN CHILDREN'S REPORTS OF PARENTAL SOCIOECONOMIC STATUS}

The measurement reliability of socioeconomic characteristics is important in understanding the socioeconomic attainment process. Survey respondents make errors in reporting their own and others' socioeconomic statuses, reporting errors may be nonrandom, and groups may differ in the extent and pattern of their errors. Therefore, inferences based on attainment models depend on whether such models explicitly take account of measurement error (Bielby and Hauser, 1977a; Bielby et al., 1977a, 1977b; Broom et al., 1978).

Problems of measurement error in achievement process studies are more serious when children are the survey respondents who supply information on parental socioeconomic characteristics. This is typically the case in studies of the early achievement process. Because knowledge of social statuses is acquired through socialization, young persons may have a seriously incomplete picture of their parents' social standing and, therefore, make considerable errors in reporting their parents' characteristics (W. M. Mason et al., 1976, and references cited therein). The reliability of children's responses about parental characteristics varies with age. For whites, W. M. Mason et al. (1976) show that elementary school children are noticeably less reliable than their parents themselves, while high school youths approach their parents in reporting accuracy. Children's reporting errors may be nonrandom and the incidence of nonrandomness may depend on the particular parental socioeconomic characteristic for which information is elicited. The same authors also show that white elementary school children's response errors for fathers' and mothers' grades of school completed are positively correlated, while reporting errors for other pairs of parental statuses for elementary school children and for all pairs of statuses for older children are essentially random.

These findings on the quality of children's reports by grade level are based on measurement models estimated separately for each of three grades. As a result, the between-grade findings are 
not supported by statistical tests. In addition, separate estimation for each group results in less than optimal estimates of measurement model parameters. As will be discussed below, some parameters of the measurement models should not vary across grade levels. Estimating a separate model for each group, therefore, requires estimating more parameters than are needed, reducing the reliability of parameter estimates.

In the following discussion, we outline a measurement model for a single grade level, point out where between-group constraints can produce a parsimonious multiple group model, and discuss the simultaneous measurement model.

\section{A SINGLE GROUP MEASUREMENT MODEL}

Our data consist of parents' and children's reports on three parental socioeconomic characteristics-father's occupation, father's grades of schooling, and mother's grades of schoolinginvolving children in the sixth, ninth, and twelfth grades. ${ }^{2} \mathrm{~A}$ strategy for modeling children's response errors is to specify linear covariance structure models of parental and child reports. Parents' and children's reports of the same parental characteristics are viewed as linear functions of a common, unobservable true parental status. For each grade level, for the $i^{\text {th }}$ individual, the model can be written as follows:

$$
\begin{aligned}
& \text { FAFED }_{\mathrm{i}}=\text { TRFED }_{\mathrm{i}}+\epsilon_{1 \mathrm{i}} \\
& \text { MOMED }_{\mathrm{i}}=\text { TRMED }_{\mathrm{i}}+\epsilon_{2 \mathrm{i}} \\
& \text { FAFOC }_{\mathrm{i}}=\text { TRFOC }_{\mathrm{i}}+\epsilon_{3 \mathrm{i}} \\
& \text { SOFED }_{\mathrm{i}}=\lambda_{4} \text { TRFED }_{\mathrm{i}}+\epsilon_{4 \mathrm{i}} \\
& \text { SOMED }_{\mathrm{i}}=\lambda_{5} \text { TRMED }_{\mathrm{i}}+\epsilon_{5 \mathrm{i}} \\
& \text { SOFOC }_{\mathrm{i}}=\lambda_{6} \text { TRFOC }_{\mathrm{i}}+\epsilon_{6 \mathrm{i}} .
\end{aligned}
$$


TRFED is father's true (unobservable) grades of schooling completed, and FAFED and SOFED are father's and son's reports of father's schooling respectively. Similarly, TRMED is mother's true grades of schooling completed, and MOMED and SOMED are mother's and son's reports of mother's schooling respectively. TRFOC is father's true occupational status score, and FAFOC and SOFOC are the status scores of father's and son's reports of father's occupation. The $\lambda$ 's are parameters to be estimated from the data. The parameters $\lambda_{1}, \lambda_{2}$, and $\lambda_{3}$ have been set equal to unity in equations 1,2 , and 3 to identify the model. The $\epsilon$ 's are stochastic disturbances. All variables are expressed as deviations from their respective means.

To complete the model, we specify the pattern of covariances among the true parental characteristics and among the errors in the six equations. True scores are allowed to covary freely, providing estimates of the covariances among parental characteristics corrected for measurement error. Error covariances are specified as free or zero, depending upon the extent to which respondents use information on one status characteristic in reporting another.

Thus we have a six-equation measurement model for each grade level. The three coefficients relating sons' reports to their respective true parental statuses, the six elements of the covariance matrix of the true parental statuses, and the variable number of elements of the covariance matrix of the errors are estimated by the method of maximum likelihood. The model's adequacy in reproducing the observed covariance matrix of parents' and children's reports is evaluated by a goodness-of-fit statistic (Jöreskog, 1969). The extent of nonrandom measurement error is given by the model itself (that is, by the between-equation covariances of $\epsilon$ 's). The reliabilities of the reports are calculated from the parameter estimates. For example, consider fathers' and sons' reports of fathers' schooling (FAFED and SOFED). Let $\hat{\phi}_{11}$ be the estimated variance of TRFED, $\hat{\psi}_{11}$ be the estimated variance of the disturbance $\epsilon_{1}$, and $\hat{\psi}_{44}$ be the estimated variance of the disturbance $\epsilon_{4}$. Then the estimated reliability of FAFED is

$$
\frac{\hat{\phi}_{11}}{\hat{\phi}_{11}+\hat{\psi}_{11}}
$$


and the estimated reliability of SOFED is

$$
\frac{\hat{\lambda}_{4}^{2} \hat{\phi}_{11}}{\hat{\lambda}_{4}^{2} \hat{\phi}_{11}+\hat{\psi}_{44}}
$$

(Joreskog, 1971b). That is, an indicator's reliability is the fraction of its expected variance due to the variance of its corresponding true score.

Following our earlier discussion, this model has several limitations. First, comparisons across grade levels can be casual at best, as estimating the models independently affords no formal way of testing between-grade differences. Second, between-grade comparisons of children's reporting reliabilities are affected by extraneous sources of grade-to-grade variation. The reliability of children's reports cannot be assessed independently of the true parental status distributions and parents' reports of their statuses. Both the true characteristics and parents' reports of them, however, vary across grades due to sampling variability. Therefore, parameter estimates describing the relationship between true parental statuses and children's reports of themand thus the estimated child report reliabilities-may vary independently of the effects of maturation. Fluctuations in the quality of parental reports need not induce systematic biases in the reliability estimates for children, but they do reduce the precision of the reliability estimates.

Finally, estimating separate models for each group, results in unnecessary complexity. As discussed below, one can combine the information for all grades to estimate a single set of true parental status covariances and parental report reliabilities. This relatively parsimonious formulation fits the data at hand. Estimating a separate model for each group, therefore, requires many more parameters than are needed to describe the data.

\section{A MULTIPLE GROUP MEASUREMENT MODEL}

Superior reliability estimates of parents' and children's reports and explicit statistical tests of between-grade measurement model differences can be obtained using a framework which simultaneously estimates the measurement parameters for all 
grade levels. In the model described above, separate sets of parameters for covariances among true parental characteristics and for the error variances of parental reports are estimated for each group. In general, therefore, the estimated reliabilities of parental reports will vary over children's grade levels. But while children's reporting performance can be expected to vary with their ages (as a result of the learning process), parents' performances should not depend upon children's ages. Nor should the joint distribution of true parental characteristics vary by children's ages. ${ }^{3}$ Accordingly, we seek a model which allows the parameters reflecting children's reporting to vary by age, but which constrains the parental performances and status distributions to be stable over children's ages. In particular, we wish to modify the model described above by constraining the true status covariance matrix and the disturbance variances for parental reports to be invariant across grade levels. Such models may be estimated in Jöreskog's (1971a) general framework for the simultaneous covariance structure analysis of multiple populations. Within this framework it is possible to specify both group-invariant and group-specific parameters and to assess the adequacy of interand intragroup parameter restrictions through goodness-of-fit statistics.

Jöreskog's model is applied to our measurement error problem as follows: We estimate equations 1 through 6 above for each of three populations. Suppressing the individual level subscript $i$, we can express these equations in the matrix form

$$
X_{J}=\Lambda, F_{J}+\epsilon_{j}
$$

where $X_{j}$ is a $6 \times 1$ vector of observed parental and child reports expressed as deviations from their means, $\Lambda_{\mathrm{J}}$ is a $6 \times 3$ matrix of coefficients, $F_{j}$ is a $3 \times 1$ vector of true parental status variables, $\epsilon_{1}$ is a $6 \times 1$ vector of disturbances, and $j=1,2,3$. The covariance matrix for the status reports implied by the model is then

$$
\&\left(X_{j} X_{j}\right)=\Lambda_{j} \Phi_{j} \Lambda_{j}^{\prime}+\Psi_{j},
$$

where $\Phi_{j}=\&\left(F_{j} F_{j}^{\prime}\right), \Psi_{j}=\&\left(\epsilon_{j} \epsilon_{j}^{\prime}\right)$, and $\&$ denotes the expectation operator. When we stipulate that the joint distribution of true 
parental statuses and the reliability of parents' reports are invariant, we require that $\Phi_{1}=\Phi_{2}=\Phi_{3}$, and

$$
\psi_{(i \mathrm{i}) 1}=\psi_{(\mathrm{i}) 2}=\psi_{(\mathrm{i}) 3} \quad(\mathrm{i}=1,2,3),
$$

where the last subscript of $\psi$ refers to grade level and the (ii) denotes the first three diagonal elements of $\Psi_{j}$, that is, the error variances of the equations for parents' reports of the three parental status characteristics. Equating the true score variances and the disturbance variances for parents' reports across groups guarantees, by the definition of reliability (equation 7), that parental report reliabilities are group invariant. ${ }^{4}$ To complete the model, the form of the off-diagonal elements of $\Psi_{j}$, the error covariance matrix for each grade level, must be specified. We consider the form of $\Psi_{j}$ below.

\section{SPECIFICATIONS AND HYPOTHESES}

This section discusses the range of measurement model specifications possible within the framework outlined above and presents hypotheses to guide the interpretation of the empirical results. Equations 1 through 6 show parents' and sons' reports as linear functions of unmeasured true parental statuses. Equation 10 expresses the covariances of the status reports implied by the model as functions of true status and disturbance covariances. Equation 11 states the between-group equality restrictions on the true parental characteristics and the error variances of parents' reports. It remains to specify the pattern of parents' and sons' measurement errors. We must determine whether the model implies that reports of parental status characteristics are independent or whether some of the reports depend upon one another.

To specify whether reports depend only upon the true status they represent or upon reports of other status characteristics as well is to set the form of the disturbance covariance matrix $\Psi_{j}$. The general form for the $\mathrm{j}^{\text {th }}$ grade group is given in Figure 1. Because $\Psi_{\mathrm{j}}$ is symmetric, it can be partitioned into three distinct $3 \times 3$ submatrices of disturbance covariances: $\Psi_{11}$ for parents' 


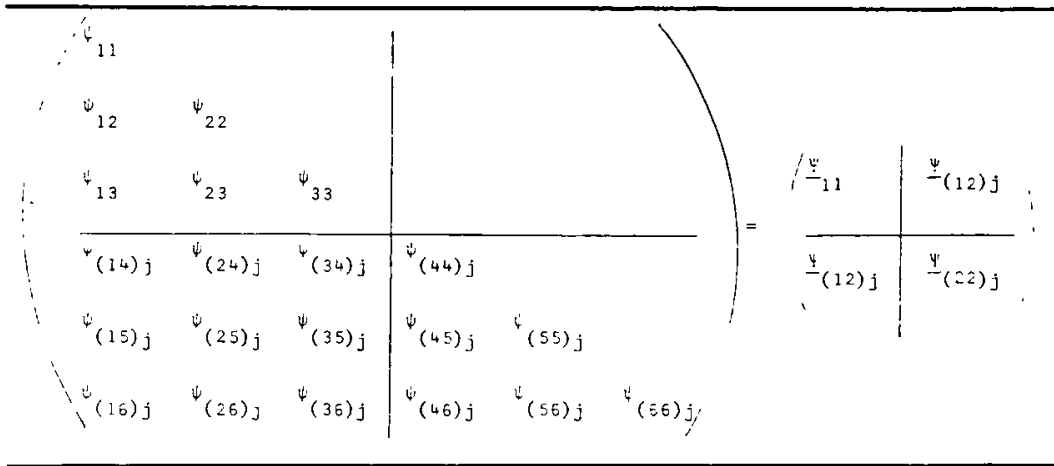

Figure 1: General form of the disturbance covariance matrix for the $j^{\text {th }}$ grade level $\left(\psi_{\mathrm{j}}\right)$.

reports, $\Psi_{(22) \mathrm{j}}$ for sons' reports, and $\Psi_{(12) \mathrm{j}}$ between parents' and sons' reports. The j subscript is not used with $\Psi_{11}$, as the covariances among parents' reports do not vary across grade levels of their sons. The elements of $\Psi_{(22) \mathrm{j}}$ and $\Psi_{(12) j}$, on the other hand, are indexed by sons' grades, to indicate that the pattern of error covariances may be age-dependent.

For each group there are a number of possible error patterns. The simplest is random error. In this case, $\Psi_{j}$ is diagonal for each grade level, where the diagonal elements are the error variances of sons' and parents' reports. If errors are not random, then it is necessary to consider several forms of nonrandomness. One form is nonrandomness among sons' reports, implying nonzero covariances among the errors in sons' reports; that is, nonzero off-diagonal elements in submatrix $\Psi_{(22) \mathrm{j}}$. In general, $\Psi_{(22) \mathrm{j}}$ is not the same for all $\mathrm{j}$, as patterns of nonrandomness among sons' reports will vary by grade level. A second form is nonrandomness among parents' own status reports. This implies nonzero offdiagonal elements in submatrix $\Psi_{11}$ of Figure 1. In contrast to the error covariances for sons' reports, those for parents' reports should not vary across sons' grade levels. The third type of nonrandom disturbance is between parents' and sons' reports. Nonzero covariances between the disturbances of parents' and sons' reports imply nonzero elements in submatrix $\Psi_{(12) j}$ in Figure 1. This class of disturbance covariances, while logically possible, is of minimal substantive interest here. It is difficult 
to conceive of mechanisms generating covariances between parents' and sons' reporting errors.

A second range of possibilities reflects alternative assumptions about children's changing report reliability across grade levels. Although parental reporting reliability and status distributions are assumed constant across grade levels from the outset, sons' report reliabilities are initially assumed to vary. In some cases, however, estimates of sons' reliabilities may turn out to be essentially unchanging across grades, even though we have not assumed this in advance. In addition, for some grade levels, sons' report reliabilities may turn out not to differ from those of their parents (W. M. Mason et al., 1976). In such cases it is desirable to reestimate models which explicitly incorporate these findings.

To do so requires constraints on the elements of $\Lambda_{j}$ and $\Psi_{j}$. Consider the covariance matrix $\Psi_{\mathrm{j}}$ in Figure 1, along with the coefficient matrix of the $\mathrm{j}^{\text {th }}$ group:

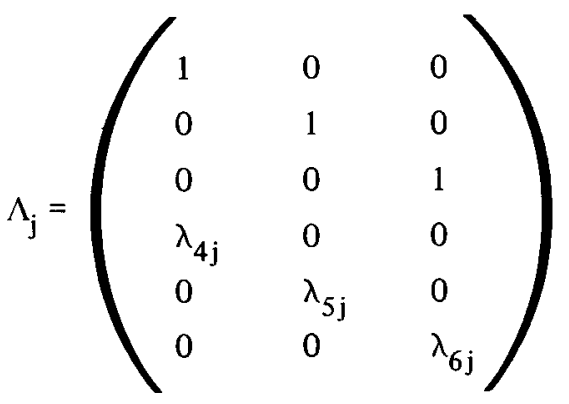

Insofar as we wish to allow the reliability of sons' reports to vary across grade levels, we place no restrictions on the elements of $\Lambda_{j}$ and $\Psi_{j}$. But if, for example, we want to specify a priori that the reliability of sons' reports of fathers' occupations for ninth- and twelfth-grade sons are equal, we impose the constraints $\lambda_{62}=\lambda_{63}$ and $\psi_{(66) 2}=\psi_{(66) 3}$. If we want to specify a priori that twelfth-grade sons report mothers' schooling as reliably as their mothers, then we impose the constraints $\lambda_{53}=1.0$ and $\psi_{(55) 3}=\psi_{22}$.

There are many possible specifications, and substantive reasoning is required to select a reasonable class of models from 
which to seek a model that fits the data. Such reasoning is developed at length by W. M. Mason et al. (1976) and is briefly summarized below. The discussion is organized around the hypothesis that reporting reliability and the incidence of nonrandom reporting errors vary by sons' ages and the parental status being reported.

\section{VARIATIONS IN RELIABILITY BY AGE}

Boys in lower grades are more likely than boys in higher grades to report their parents' statuses with error. As boys grow older their sensitivity to dimensions of social stratification increases. Older boys, moreover, will simply have heard more often what their parents' educational attainments and occupations are. At some point, therefore, sons' and parents' reporting reliability can be expected to converge.

\section{VARIATIONS IN RELIABILITY}

BY PARENTAL CHARACTERISTICS

We seek reliability estimates for reports of three parental statuses: mother's and father's grades of school completed, and father's occupation. Children may not learn these three characteristics at the same rate, implying that their report reliability will vary by the characteristics that they are asked about. In contrast with father's occupation, which is current and tangible, parental education is remote and relatively abstract. Children may frequently hear about the kind of work their fathers do, but possibly less often about how far their parents went in school. Therefore, sons may be better reporters of their father's occupation than of the educational attainment of either parent, and may approach their parents' report reliability for father's occupation at a lower grade level than for their parents' educational attainments.

Respondents uncertain about parental characteristics they are asked to report may use their knowledge of other statuses to 
help them. Operationally, this implies nonzero covariances between the errors in respondents' reports. If nonrandom reporting errors result from uncertainty, then the least knowledgeable respondents-boys in the lowest grades-will be most likely to have nonzero error covariances.

\section{VARIATIONS IN NONRANDOMNESS} BY PARENTAL CHARACTERISTICS

Not all reporting errors, however, are equally likely to be nonrandomly distributed. Mother's and father's grades of schooling are reported in response to a common stimulus, that is, a request for the highest grade of school completed by each parent, and sons therefore may resolve their uncertainty by making these two measures agree. Such agreement results, then, from a common basis of measurement for the variables, rather than from a sophisticated appreciation of assortative mating. Conversely, other types of nonrandom error are less common because they presuppose too sophisticated an understanding of social stratification. A boy uncertain of his father's schooling might guess it from his father's occupation. But the sons most likely to be uncertain of their parent's statuses-the youngest-are least likely to have the understanding to make informed guesses. Such sophisticated forms of nonrandom error therefore are unlikely to occur.

\section{EMPIRICAL RESULTS}

This section presents the results of our measurement error model estimation for young white males. We will first present summary goodness-of-fit statistics for a number of specifications. Then we will discuss the parameter and reliability estimates for the model we deem best, interpreting these results in light of the arguments presented above.

Several criteria, in addition to goodness-of-fit, are used to select a "best" model. First, parameter estimates should imply that both parents and sons report parental status with at least some error. Although equations 1 through 6 of the model require 
this, some empirical versions of the model may imply zero or negative error variances, suggesting that the model is misspecified. Second, the parameter estimates should imply reliability estimates for sons' reports which are stable or increasing with grade level. If estimates imply that sons' reporting performances deteriorate with age, this suggests either an incorrectly specified pattern of error covariances or implausible estimates resulting from sampling variability. In the latter case, a specification which equates sons' report reliabilities over grade levels is in order. Third, parents' own status reports should be at least as reliable as their sons' reports. And finally, the error covariance pattern must be substantively interpretable.

In selecting a measurement model we sought a plausible pattern of error covariances which fits the data, and then, given

TABLE 1

Goodness-of-Fit Statistics for Measurement Models for Sixth, Ninth, and Twelfth Grade White Males

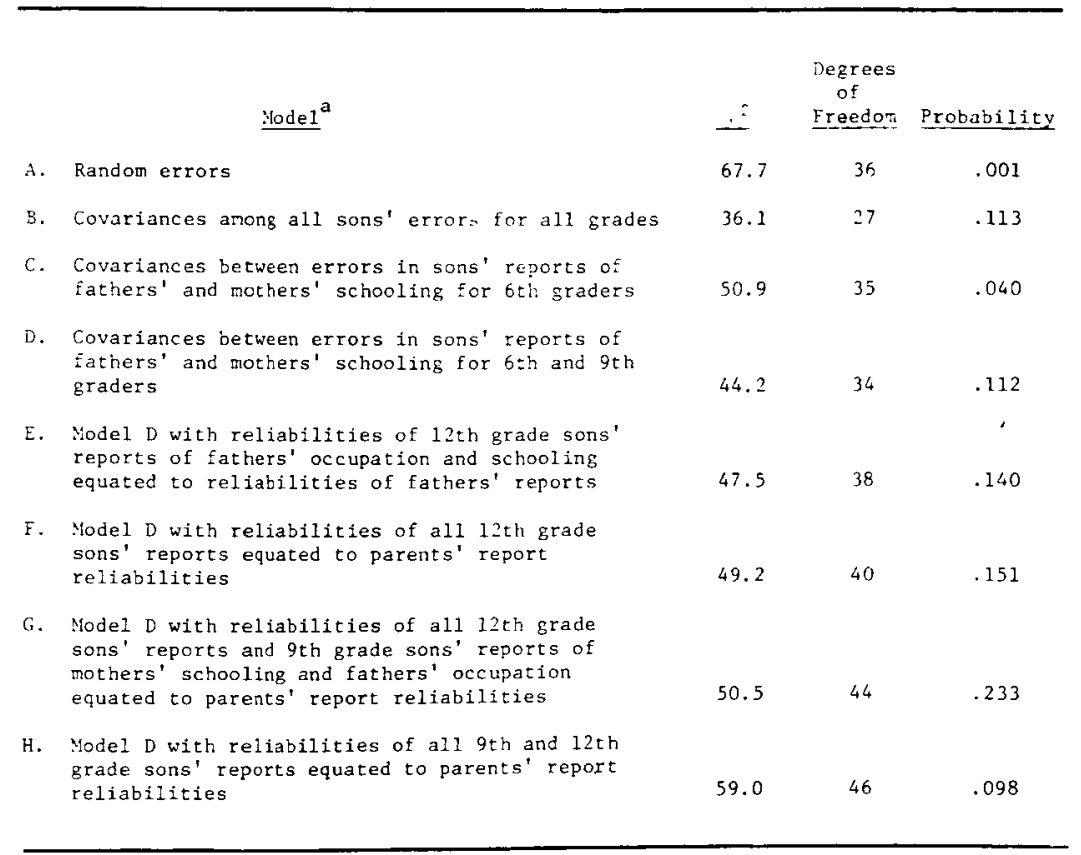

a. In all models the covariance matrix of true parental characteristics and the error variances of parents' reports are held constant across grades. 


\section{TABLE 2}

Estimated Reliabilities of Sons' and Parents' Reports of Parental Socioeconomic Characteristics, by Grade

\begin{tabular}{|c|c|c|c|c|}
\hline \multirow[b]{2}{*}{ Characteristic } & \multirow[b]{2}{*}{ Reporter } & \multicolumn{3}{|c|}{ Grade of Son } \\
\hline & & $\underline{6}$ & $\underline{9}$ & 12 \\
\hline Father's Schooling & $\begin{array}{l}\text { Father } \\
\text { Son }\end{array}$ & $\begin{array}{l}.853 \\
.611\end{array}$ & $\begin{array}{l}.853 \\
.760\end{array}$ & $\begin{array}{l}.853 \\
.944\end{array}$ \\
\hline Mother's Schooling & $\begin{array}{l}\text { Mother } \\
\text { Son }\end{array}$ & $\begin{array}{l}.890 \\
.498\end{array}$ & $\begin{array}{l}.890 \\
.862\end{array}$ & $\begin{array}{l}.890 \\
.870\end{array}$ \\
\hline Father's Occupation & $\begin{array}{l}\text { Father } \\
\text { Son }\end{array}$ & $\begin{array}{l}.915 \\
.710\end{array}$ & $\begin{array}{l}.915 \\
.908\end{array}$ & $\begin{array}{l}.915 \\
.937\end{array}$ \\
\hline
\end{tabular}

NOTE: The model includes error covariances between sons' reports of father's and mother's schooling for sixth and ninth grade white males (Model $D$ in Table 1).

this pattern, considered alternative sets of between-grade reliability constraints to find the point at which the boys' responses are as reliable as their parents'.

Table 1 presents goodness-of-fit statistics for eight distinct specifications. ${ }^{5}$ Each of these specifications requires the estimation of equations 1 through 6 simultaneously for sixth-, ninth-, and twelfth-grade boys. In addition, each specification incorporates the intergrade equality restrictions for parents discussed above: to wit, the covariance matrix of the unobservable true parental status reports and the error variances of parental reports of their socioeconomic characteristics are held constant across sons' grade levels.

Model A of Table 1 assumes that all reporting errors are random. The likelihood ratio chi-square value for this model is 67.7 with 36 degrees of freedom, indicating that random reporting errors are very unlikely to have generated the observed covariance matrices. In addition, this specification implies implausible reliability estimates (not shown here). All reliabilities for twelfth-grade sons' reports and one reliability for ninth-grade sons' reports exceed the corresponding parents' reliabilities. Evidently a more complex model is required. 
TABLE 3

Model G Parameter Estimates

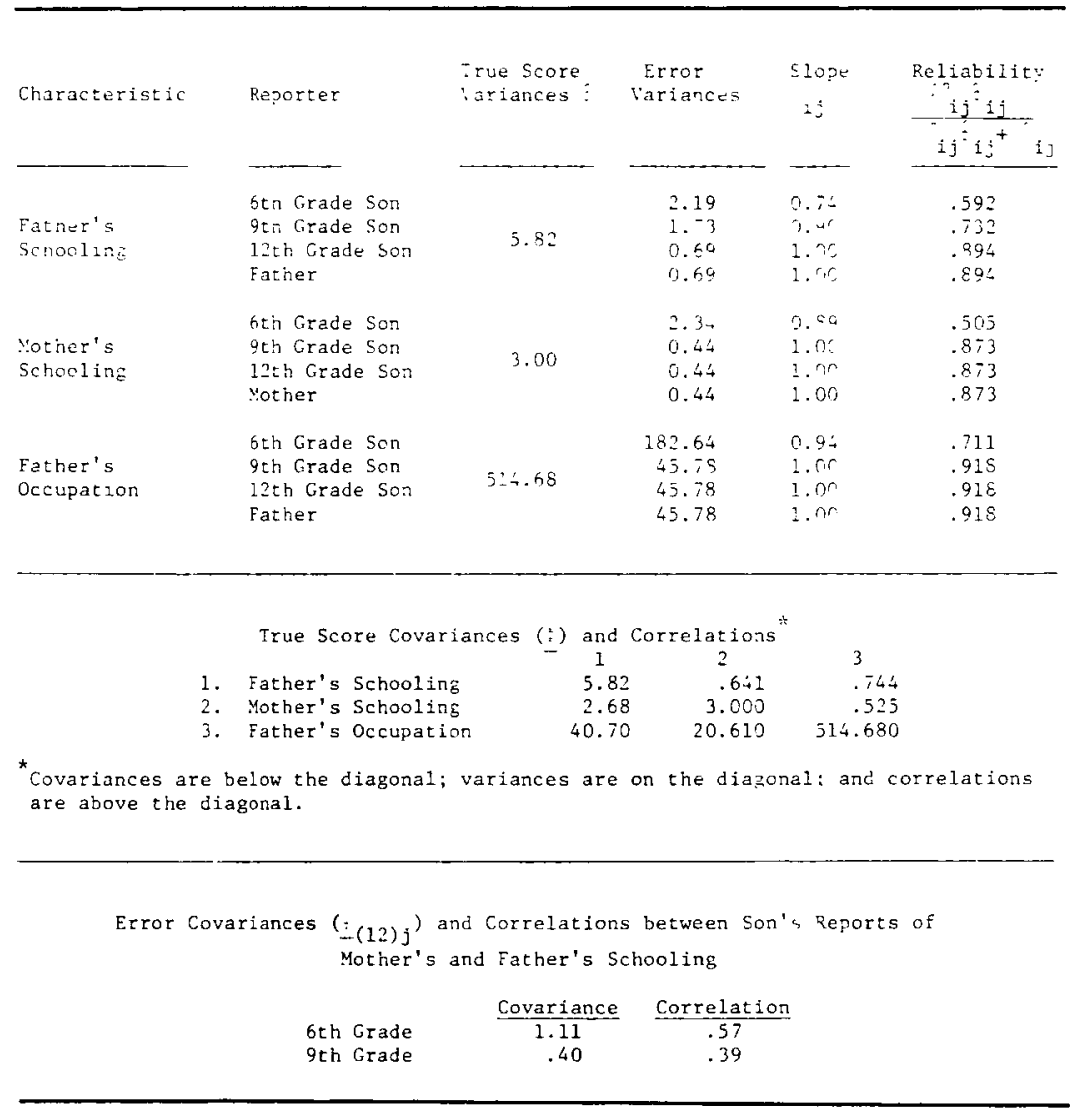

Previous analysis of these data indicates that the only significant correlation in the errors of sons' reports is for the reports of mothers' and fathers' schooling for sixth-graders (W. M. Mason et al., 1976). To see if a similar finding results from the present estimation framework, we first consider a less parsimonious model in which all sons' errors are mutually correlated (Model B in Table 1). Both the sizes of the error correlations implied by the estimated error covariances and their standard errors will suggest which error covariances should be retained in the model. With a loss of nine degrees of freedom 
TABLE 4

Covariance Matrices for White Sixth, Ninth, and Twelfth Grade Sons' and Parents' Reports of Parental Socioeconomic Characteristics

\begin{tabular}{|c|c|c|c|c|c|c|}
\hline & 1. & $\underline{2}$ & 3. & 4. & $\underline{5 .}$ & 6. \\
\hline \multicolumn{7}{|c|}{ Sixth Grade } \\
\hline 1. SOFED & 5.86 & & & & & \\
\hline 2. SOMED & 3.12 & 3.32 & & & & \\
\hline 3. SOFOC & 35.28 & 23.85 & 622.09 & & & \\
\hline 4. FAFED & 4.02 & 2.14 & 29.42 & 5.33 & & \\
\hline 5. YONED & 2.99 & 2.55 & 19.20 & 3.17 & 4.64 & \\
\hline 6. FAFOC & 35.30 & 26.91 & 465.62 & 31.22 & 23.38 & 546.01 \\
\hline \multicolumn{7}{|c|}{ Ninth Crade } \\
\hline 1. SOFED & 8.20 & & & & & \\
\hline 2 SO:SLD & 3.47 & 4.36 & & & & \\
\hline 3. SOEOC & 45.65 & 22.58 & 611.63 & & & \\
\hline 4. FAFED & 6.39 & 3.16 & 44.62 & 7.32 & & \\
\hline 5. YOYED & 3.22 & 3.77 & 23.47 & 3.33 & 4.02 & \\
\hline 6. FAFOC & 45.58 & 22.01 & 548.00 & 40.99 & 21.43 & 585.14 \\
\hline \multicolumn{7}{|c|}{ Twelfth Grade } \\
\hline 1. SOFED & 5.74 & & & & & \\
\hline 2. SOMED & 1.35 & 2.49 & & & & \\
\hline 3. SOFOC & 30.24 & 12.73 & 535.30 & & & \\
\hline 4. FAFED & 4.94 & 1.65 & 37.36 & 5.39 & & \\
\hline 5. YOMED & 1.67 & 2.32 & 15.71 & 1.85 & 3.06 & \\
\hline h. FOFOC & 40.11 & 12.94 & 496.86 & 38.09 & 24.91 & 538.76 \\
\hline
\end{tabular}

NOTE: SOFED = son's report of father's schooling, SOMED = son's report of mother's schooling, SOFOC = son's report of father's occupation, FAFED = father's report of his schooling, MOMED $=$ mother's report of her schooling, FAFOC $=$ father's report of his occupation. The number of observations is 80 for each group.

(one for each error covariance in each of the three grade levels), the chi-square statistic drops by more than thirty, a highly significant improvement in fit. The standard errors of the error covariances (not shown here), however, indicate that all but two of the nine covariances are not significantly different from zero. These are the covariances between the sons' errors in reporting fathers' and mothers' schooling for both sixth- and ninth-graders.

Models $\mathrm{C}$ and $\mathrm{D}$ in Table $\mathrm{l}$ are suggested by the above findings. The first assumes a single error covariance between sons' reports of parents' educational attainments for sixth-graders, the model implied by the earlier analysis. The second assumes that the error covariances between sons' reports of parents' schooling occur for both sixth- and ninth-grade sons. Plainly, the latter 
has a superior fit. Model $\mathrm{C}$ is a clear improvement upon the purely random error model, but, for the additional degree of freedom required to estimate Model $\mathrm{D}$, the chi-square statistic drops by more than six points. The descriptive levels of significance in the last column of the table show, moreover, that Model $\mathrm{D}$ is as likely to have generated the sample covariances as Model B, in which all sons' disturbances are correlated.

We next consider whether Model D implies reasonable estimates of sons' and parents' report reliabilities. Table 2 presents reliabilities calculated from the parameter estimates of the model. For each of the three parental socioeconomic characteristics, sons' reporting reliabilities increase monotonically with grade level. These estimates imply, however, that for both fathers' characteristics, twelfth-grade sons are more reliable respondents than their fathers. To see if these anomalies result from sampling fluctuations, we can estimate a more constrained model which retains the present pattern of disturbance covariances, but equates the reliabilities for sons' and parents' reports. If these constraints do not make the model fit significantly worse than Model $\mathrm{D}$, then we have adequately characterized the data.

In addition to rectifying the anomalously high sons' reliabilities in Table 2, we also want to see the point at which sons' and parents' reports are equally accurate. Table 2 suggests that not only twelfth-grade sons' reports of fathers' statuses, but also their reports of mothers' schooling and at least some of the ninth-graders' reports may be as reliable as the corresponding parents' reports. Hence we estimate a variety of models, making differing assumptions about the point at which sons' and parents' reports are equally reliable.

Goodness-of-fit measures for alternative between-grade equality specifications appear in Table 1 (Models E, F, G, and $\mathrm{H}$ ). Model E, which has equal parent and son reliabilities for twelfthgraders' reports of fathers' characteristics, preserves the satisfactory fit of Model D. Models $F$ and $G$ further improve the specification through additional equality constraints between sons' and parents' report reliabilities. The excellent fit of Model $G$ suggests that twelfth-graders report all three parental statuses as reliably as parents, and that ninth-graders report both mothers' schooling and fathers' occupation as well as the parents. If we go 
on to Model $\mathrm{H}$, however, and equate ninth-grade sons' and their fathers' reliabilities for fathers' schooling, the fit of the model deteriorates markedly.

The analysis suggests that children do not approach their parents' level of reporting accuracy at the same rate for all characteristics, but by the twelfth grade, sons and parents are equally reliable for all characteristics we consider. Parameter and reliability estimates for Model G, which embodies these findings, appear in Table 3. The reliability estimates for parents' and sons' reports given in the final column of the table confirm several of the arguments made above. First, they show that sixth-graders are only one-half to two-thirds as reliable respondents as twelfthgraders and their parents. Second, the estimates show that boys report father's occupation more accurately than the schooling of either parent. These differences occur at every grade level, though they are generally weaker at the higher grade levels. Finally, white boys do not favor either parent in reporting grades of schooling. Father's schooling is reported somewhat more reliably than mother's by sixth- and twelfth-graders, but the opposite is true for ninth-graders.

The final panel of Table 3 presents the estimated covariances and correlations between errors in reports of mothers' and fathers' schooling by sixth- and ninth-grade sons. These correlations are substantial, suggesting that sons may resolve their uncertainty about parental schooling by reconciling their parents' schooling levels, two variables which are measured in the same metric.

\section{CONCLUSION}

This article has illustrated the use of the simultaneous factor analytic methods developed by Jöreskog (1971a) in estimating measurement models for children's reports of parental socioeconomic characteristics at various grade levels. By estimating models for all grade levels simultaneously, we have used the structure of the data to obtain estimates superior to those obtainable from estimating separate models for each group. In partic- 
ular, we have held equal those parameters which should be naturally stable across groups (except due to sampling variability). This produces a more parsimonious and reliable model for the several groups taken together. In addition, the simultaneous estimation permits explicit statistical tests of group differences in measurement error patterns, permitting further model simplification.

\section{NOTES}

1. Since this article was written, software has become available for estimating simultaneous equation models with both observed and unobserved variables in multiple populations (Jöreskog and Sörbom, 1978). Although these models are more complex than the ones discussed in this article, their logic and rationale are similar.

2. For a full description of the sample design and the complete data set, see Kerckhoff (1974). The data derive from a 1969 sample of approximately 500 Fort Wayne, Indiana black and white sixth-, ninth-, and twelfth-grade boys and their parents; however, the present analysis is based on the white sample. The boys and their parents reported parental statuses in independent interviews. Parents' schooling is measured in grades completed, and father's occupation is measured by the Duncan socioeconomic index. Table 4 presents the covariance matrices of children's and parents' reports for the 3 grade levels used in the analysis. Covariances are pairwise present, that is, calculated over all nonmissing observations on each pair of variables. Variances are based on the maximum numbers of nonmissing univariate observations.

3. In principle, there may be slight nonsampling variability among parents by children's ages in cross-sectional data. On average, parents of older children are older than parents of younger children and may therefore have somewhat different status distributions insofar as socioeconomic characteristics are related to age. Such variations, however, should be very small. In these data the most extreme age difference between groups of children averages six years. A corresponding six-year age difference for their parents implies trivial differences in their status distributions. Moreover, neither in the raw data nor in the earlier analyses of W. M. Mason et al. (1976) is there evidence of systematic variation in parental status distributions or parental report reliability by children's grade level.

4. The converse, however, is not true. Equal reliability requires only that the ratio of the true score variance to the sum of the true score variance and the report error variance be equal, rather than the variances themselves. The restrictions given by equation 11 , then, are stronger than a strict equal-reliability condition requires. We impose these restrictions for two reasons. First, they are not unreasonable. Neither the distributions of true parental characteristics nor the parental reports' error variances should vary significantly by children's grade level. Second, the equal-reliability restriction per se is nonlinear (see equation 7), and therefore difficult to impose in practice.

5. All models were estimated using the program SIFASP (van Thillo and Jöreskog, 1970). 


\section{REFERENCES}

BIELBY, W. T. and R. M. HAUSER (1977a) "Response error in earnings functions for nonblack males." Soc. Methods and Research 6 (November): 241-280.

(1977b) "Structural equation models." Annual Rev. of Sociology 3: 137-161.

BIELBY, W. T., R. M. HAUSER, and D. L. FEATHER MAN (1977a) "Response errors of black and nonblack males in models of the intergenerational transmission of socioeconomic status." Amer. J. of Sociology 82 (May): 1242-1288.

- - (1977b) "Response errors of nonblack males in models of the stratification process." J. of Amer. Stat. Assn. 72 (December): 723-735.

BROOM, L., F. L. JONES, P. McDONNELL, and P. DUNCAN-JONES (1978) "Is it true what they say about daddy?" Amer. J. of Sociology 84 (September): 417-426.

GOLDBERGER, A. S. and O. D. DUNCAN (1973) Structural Equation Models in the Social Sciences. New York: Seminar.

JORESKOG, K. G. (197/a) "Simultaneous factor analysis in several populations." Psychometrika 36 (December): 409-426.

(1971 b) "Statistical analysis of sets of congeneric tests." Psychometrika 36(June): 109-133.

- _ (1969) "A general approach to confirmatory maximum likelihood factor analysis." Psychometrika 34 (June): 183-202.

-_- and D. SOR BOM (1978) LISREL IV: Analysis of Linear Structural Relationships by the Method of Maximum Likelihood. Chicago: National Educational Resources.

KERCK HOFF, A. C. (1974) Ambition and Attainment. Washington, DC: American Sociological Association.

MASON, K. O., J. L. CZAJKA, and S. ARBER (1976) "Change in U.S. women's sexrole attitudes, 1964-1974." Amer. Soc. Rev. 41 (August): 573-596.

MASON, W. M., R. M. HAUSER, A. C. KERCKHOFF, S. S. POSS, and K. MANTON (1976) "Models of response-error in student reports of parental socioeconomic characteristics," pp. 443-494 in W. H. Sewell et al. (eds.) Schooling and Achievement in American Society. New York: Academic.

van THILLO, M. and K. G. JORESKOG (1970) SIFASP-A General Computer Program for Simultaneous Factor Analysis in Several Populations. Research Bulletin 70-62. Princeton, NJ: Educational Testing Service.

Robert D. Mare is Assistant Professor of Sociology at University of WisconsinMadison. He is completing a monograph on changes in educational stratification in the United States and is conducting research on recent changes in the youth labor force and on socioeconomic influences on child mortality.

William M. Mason is Associate Professor of Sociology and Associate Research Scientist of the Population Studies Center at University of Michigan. His current research includes the study of political alienation, comparative analysis of human reproductive behavior, the statistics and methodology of multilevel analysis, and methodological and substantive issues involving cohort analysis. 